\begin{tabular}{|c|c|c|}
\hline Capability & Opportunity & Motivation \\
\hline Psychological & Social & Reflective \\
\hline - Parent & - Parent & - Parent \\
\hline o Cognitive Function & oStigma & o Negative Attitudes \\
\hline \multirow[t]{3}{*}{ o Education Level } & ○ Religious/cultural beliefs & o Treatment Concerns \\
\hline & olncome & ○ Alternative Therapies \\
\hline & & - Outcome Expectations \\
\hline Physical & Physical & Automatic \\
\hline - Child & - Both & - $\underline{\text { Both }}$ \\
\hline oDrug Ingestion & o Interrupted access to health care facilities & ○ Fear and Anxiety \\
\hline o Parent & o Inconsistent doctor-patient relationship & \\
\hline o Forgetfulness & - Cost of medication & \\
\hline
\end{tabular}

Conclusions This study's main application lies in formulating interventions related to acknowledging misconceptions and stigmas related to Epilepsy and improving health-care systems by creating a team-based approach to patient care in meeting the required demands. These interventions may also be linked to devise applicable policies to impact reforming target behavior significantly.

\section{GENOTYPE/PHENOTYPE CORRELATIONS IN 125 CHINESE PATIENTS WITH TUBEROUS SCLEROSIS: A 29 YEARS' EXPERIENCE IN HONG KONG}

Samuel Yan Lik NG, Ivan FM Lo, Ho-Ming Luk. Hong Kong

\subsection{6/bmjpo-2021-RCPCH.129}

Background Tuberous Sclerosis Complex (TSC) is a multisystemic neurocutaneous disorder of autosomal dominant inheritance, with characteristic presentation of benign hamartomatous lesions across the brain, kidney, heart, eyes, skin and lungs.

Objectives To study the mutation spectrum and phenotypic characteristics of TSC patients in the Chinese population, as well as to delineate the underlying genotype-phenotype correlations and compare with previous studies.

Methods 120 patients suspected of TSC were referred to the Clinical Genetic Service (CGS), Department of Health, HKSAR for evaluation between 9/1991 and 8/2020. Blood samples from 105 patients presented with $\geq 1$ major/ $\geq 2$ minor diagnostic features according to the latest diagnostic criteria $[1,2]$ are taken. Comprehensive genetic testing including sequencing and Multi-ligand probe-dependent Amplification (MLPA) was performed. Family screening was performed on molecularly confirmed cases. In total, 133 patients had a definite diagnosis, in which the 125 Chinese patients are included into the study. Statistical analyses (Pearson $\chi 2$ tests, Fisher exact test and ANOVA) are performed using SPSS version 26.0.

Results Pathogenic genetic alternations are identified in $72.0 \%$ patients (90/125), in which $26.4 \%$ (33/125) have TSC1 and $45.6 \%(57 / 125)$ have TSC2 mutations. 28 novel mutations are reported while familial cases account for 23.2\% (29/125). Males have significantly more subependymal nodules (47/55 vs $34 / 50 ; p=0.033)$ than females, whereas de novo cases have more cortical tubers $(69 / 82$ vs $14 / 24 ; p=0.007)$ and renal angiomyolipoma $(44 / 85$ vs $3 / 21 ; p=0.002)$ than familial cases.
TSC2 cases have more frequent mental retardation (29/42 vs $4 / 25 ; \quad \mathrm{p}<0.001)$, cardiac rhabdomyoma $(21 / 45$ vs $3 / 25$; $\mathrm{p}=0.003)$, renal angiomyolipoma $(24 / 49$ vs $5 / 27 ; \mathrm{p}=0.009)$ and facial angiofibromas $(37 / 50$ vs $16 / 31 ; p=0.039)$ than TSC1, while mutation-negative cases show less subependymal nodules $(14 / 23$ vs $65 / 77 ; p=0.015)$ than mutation-positive cases. Similarly, TSC2 cases show higher occurrence of subependymal nodules $(44 / 50$ vs $14 / 23 ; \mathrm{p}=0.012)$ than mutationnegative cases though mutation-negative patients have more frequent mental retardation $(10 / 21$ vs $4 / 25 ; \mathrm{p}=0.020)$, autism spectrum disorder $(4 / 18$ vs $0 / 23 ; \mathrm{p}=0.030)$ and renal angiomyolipoma $(12 / 23$ vs $5 / 27 ; \mathrm{p}=0.012)$ than TSC1 cases. There are no significant phenotypic differences between patients with missense and protein-truncating mutations in TSC2, while TSC2 missense mutations are associated with more mental retardation $(17 / 21$ vs $4 / 23 ; \mathrm{p}<0.001)$, cardiac rhabdomyoma $(11 / 21$ vs $2 / 24 ; p=0.001)$, renal angiomyolipoma (13/23 vs $4 /$ $24 ; p=0.004)$ and renal cysts $(6 / 23$ vs $1 / 24 ; p=0.048)$ than those with TSC1 protein-truncating mutations. Mutations in the coiled-coil region (TSC1) are significantly associated with nail abnormalities. All 14 antenatal-onset patients have cardiac rhabdomyoma. Meanwhile, they have less seizures (6/11 vs $55 / 64 ; p=0.027)$ than paediatric-onset cases but have higher frequencies of mental retardation $(5 / 11$ vs $0 / 11 ; \mathrm{p}=0.035)$ than adult-onset patients. Generally, paediatric-onset patients have more neurological manifestations, while initial presentations of adult-onset TSC are more diverse. The predictive yield of antenatal cardiac rhabdomyoma on TSC is $63.6 \%$.

Conclusions The overall phenotypic spectrum and genotypephenotype correlations in our Chinese cohort are compatible with literature. 28 novel mutations have been reported in this study.

\section{A SURVEY OF PARENTAL KNOWLEDGE, ATTITUDE AND PRACTICE OF USING CHILD CAR SEAT RESTRAINTS IN SINGAPORE, WITH A SYSTEMATIC REVIEW}

Xi Yun Ling, Alvin Jia-Hao Ngeow, Mary Grace Sy Tan, Daisy Kwai-Lin Chan. Singapore

\subsection{6/bmjpo-2021-RCPCH.130}

Background Motor vehicle accidents are associated with significant mortality and morbidity in adults and children. The annual number of accidents with injury in Singapore remained at over 7600 for the past 5 years. Child car restraints prevent 